\title{
Clinical audit on prescribing antibiotics
}

\author{
General dental practitioners' experiences of a collaborative clinical audit on antibiotic prescribing: a qualitative study
}

\section{N. A. 0. Palmer and Y. M. Dailey Br Dent J 2002; 193: 46-49}

\section{Objective}

To evaluate general dental practitioners' experiences of a multicollaborative antibiotic prescribing audit.

\section{Design}

Qualitative analysis of compulsory post-audit group report data collection forms and individual practitioners' post-audit evaluation forms.

\section{Subjects}

Information was collected from 175 general dental practitioners in the North West of England who participated in the audit.

\section{Method}

The general dental practitioners were divided into groups of 8-10 to undertake the audit. Information from compulsory post-audit group reports was transcribed and analysed. The information was categorised into a number of areas including changes in practice, patients' expectations, training and quality of service. On completion of the audit individual practitioners were asked to complete an evaluation form on the audit process.

\section{Results}

$141(80.5 \%)$ individual evaluation forms were returned. Over 90\% of GDPs felt that the audit process was easily understood and the majority of the practitioners thought the audit was worthwhile. Approximately 69\% of participants felt that the audit had helped to change their antibiotic prescribing practices. Analysis of the post-audit group report data collection forms revealed more than 100 statements. The most common areas were changes required in practice, patients' expectations, increased training and quality of service.

\section{Conclusion}

The collaborative clinical audit project was seen to be a worthwhile learning experience by the participating general dental practitioners. The audit encouraged GDPs to change their antibiotic prescribing practices and thereby improve patient care. GDPs also highlighted the need for continuing education in the prescribing of antibiotics.

\section{IN BRIEF}

- Clinical audit can alter clinical practice and improve patient care.

- Clinical audit can improve knowledge.

- Clnical audit can identify areas for further continuing professional development.

- Collaborative audit has the added benefit of peer review.

- Clinical audit should involve the whole dental team.

\section{COMMENT}

The improvement of healthcare delivery is often a process of incremental steps. Audit is a relatively recent approach to aid clinicians in the provision of services to record current practice systematically, and catalogue predefined outcomes. An audit analysis can identify areas of strengths and weaknesses to precipitate change. The audit process can be seen through a number of lenses.

Through the eyes of the service manager or commissioner, this objective approach can determine the degree of change in service provision. The authors of the current paper have already described the success of their systematic audit on antibiotic prescribing in a previous report. ${ }^{1}$ This further paper outlines the results of GDPs' opinions in participating in the audit. Qualitative (theme analysis of transcripts) and quantitative (attitude scale data) analyses were employed from individual and group reports of the participants. Results showed an overwhelming positive response to the audit process. Participants stated that they learned new information and crucially reported changes in practice. Barriers to practice change appeared to be associated with difficulties in modifying the practice systems rather than any disagreement with the principle messages of modern prescribing.

A number of features of this study are particularly interesting, as observed through the eyes of a behavioural scientist. First, the positive attitudinal response to the audit process appeared consistent with the behavioural response exhibited by the participants (reduction by $43 \%$ ). ${ }^{1}$ From the small but growing literature on persuading clinicians to change their behaviour, these results are very encouraging. ${ }^{2}$ The second feature of this study to highlight, is how this change was facilitated. An obvious candidate, to explain the improvement, was the adoption of the small working group approach. Group sizes of 8-10 professionals, attending to a common set of problems to derive solutions, can be a very affirming experience. Finally, the practice teams of the participants were infrequently consulted, indicating perhaps that a single audit process will not modify systemic elements of how a practice functions. The longer term behavioural change in the prescription of antibiotics may need to focus on broader system issues such as the practice team in order to maintain the excellent results reported in this paper.

Gerry Humphris, Reader in Clinical Psychology University of Manchester

1 Palmer N A, Dailey Y M, Martin M V. Can audit improve antibiotic prescribing in general dental practice? Br Dent J 2001, 191: 253-235.

2 Ashworth M, Armstrong D, Colwill S, Cohen A, Balazs J. Motivating general practitioners to change their prescribing: the incentive of working together. J Clin Pharm Ther 2000, 25: 119-124. 